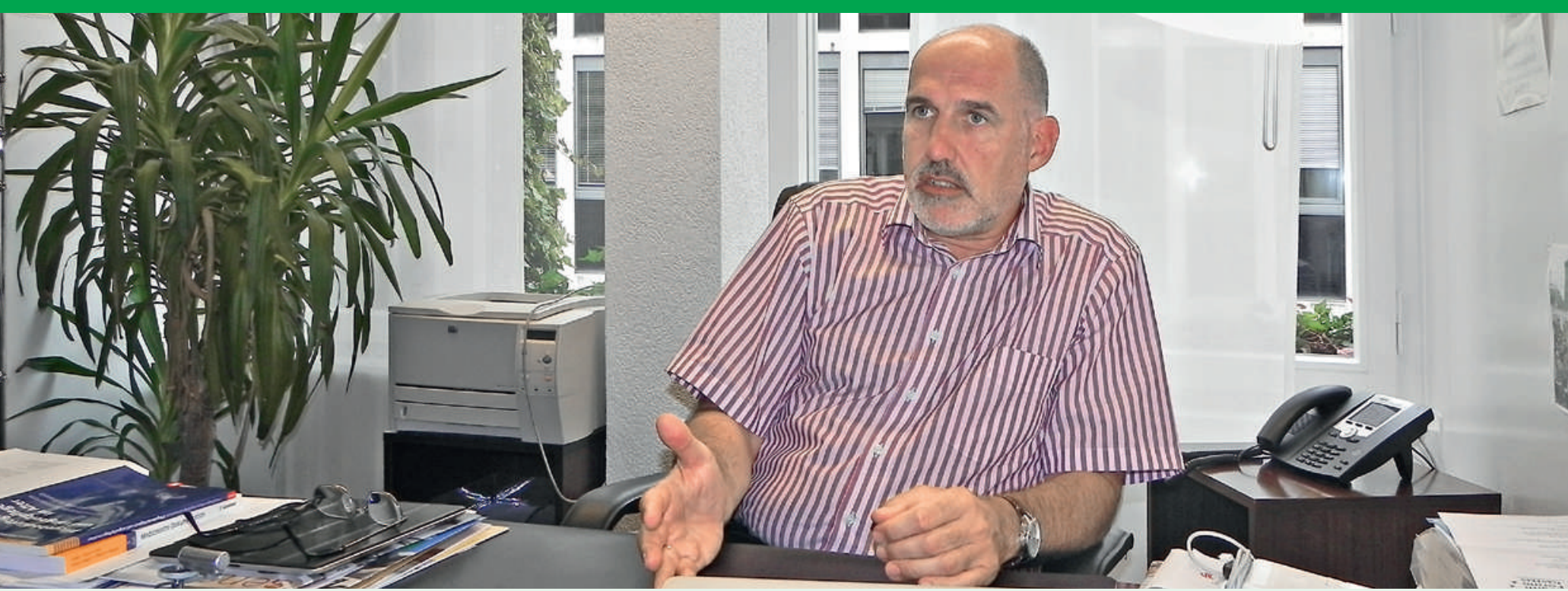

Président passionné de la FMH pendant huit ans: Jacques de Haller dans son bureau à l'Elfenstrasse à Berne.

\title{
«La FMH est toujours présente, et plus unie que jamais»
}

\author{
Pendant deux mandats, Jacques de Haller a représenté en première ligne la Fédéra- \\ tion des médecins suisses (FMH), dont il a fortement marqué la politique. Durant \\ cette interview, le président sortant établit un bilan avant de remettre les affaires à \\ son successeur Jürg Schlup* le 6 décembre prochain.
}

Interview: Bruno Kesseli

Le départ de Jacques de Haller a également inspiré la caricature d'ANNA à la dernière page de ce numéro.
* Le nouveau président de la FMH sera présenté en décembre, dans le cadre d'une interview du BMS.
Jacques de Haller, pendant les huit ans et demi de votre présidence, vous vous êtes engagé de toutes vos forces et avec passion pour votre organisation professionnelle. Vous auriez d'ailleurs volontiers continué pendant un mandat supplémentaire. Vous avez certainement été déçu que cela ne se fasse pas. Avec un recul de quelques mois, quels sont vos sentiments quand vous pensez à la Chambre médicale de juin dernier?

Jacques de Haller: Bien sûr, ne pas être réélu a constitué pour moi une certaine déception, mais ce ne fut certainement pas un drame. En réalité, je réfléchissais depuis longtemps à l'éventualité d'un troisième mandat. Au vu des nombreux projets et questions importants à l'ordre du jour pour le corps médical, j'en étais arrivé à la conclusion qu'un nouveau mandat pourrait être utile. J'ai fait ce travail pendant huit ans et lorsque je considère ces années, j'ai l'impression de l'avoir bien fait, avec des résultats positifs pour le corps médical. La Chambre médicale a pris sa décision. L’issue du vote était prévisible et ce ne fut pas une surprise.

Pourquoi avoir maintenu votre candidature en dépit de signaux d'alerte manifestes?

Un nombre important de collègues me soutenaient, et ce n'aurait pas été correct envers eux d'interrompre brutalement l'exercice et de retirer ma candidature. De plus, d'importants dossiers politiques étaient en route. Une continuité semblait judicieuse. Je rappelle que dix jours après la Chambre médicale, le référendum sur le Managed care a été remporté avec une majorité spectaculaire de $76 \%$ des voix exprimées.

L'année précédente, votre engagement politique et votre candidature au Conseil national pour le PS avaient aussi suscité des remous au sein de la FMH. Ce précédent a-t-il constitué un handicap déterminant pour votre réélection? Je ne le crois pas. Mon engagement au Parti socialiste a effectivement suscité quelques réactions lorsque les médias s'en sont emparés, mais c'est surtout ma présence au Parlement fédéral qui aurait posé des problèmes aux yeux de certains membres de la Chambre médicale... problèmes qui ne se sont finalement pas posés! En outre, ce sont essentiellement les partis de gauche qui ont soutenu les positions de la FMH ces dernières années. Plusieurs membres du Comité central élu font partie du PLR, on le sait - ils vont se trouver face au défi de convaincre des partis d'autre couleur des positions de la FMH. Et aussi de représenter à l'extérieur la multiplicité des sensibilités politiques de nos membres. 
La continuité que vous souhaitiez pour la FMH n'aura donc pas lieu...

Effectivement, les choses vont changer et la Chambre médicale en a décidé ainsi. Mais il existe toujours un important potentiel de développement. Il serait dommage que la politique et le nouveau style de la FMH ne permettent pas d'exploiter ce potentiel.

\section{«'ai fait le travail pendant huit ans et lorsque je considère ces années, j'ai l'impression de l'avoir bien fait.»}

\section{Qu'entendez-vous par là?}

L'évolution de ces dernières années est claire, nous sommes devenus l'acteur principal du système de santé. Il est à long terme décisif pour la FMH et pour le corps médical de continuer à marquer l'ensemble de la politique de la santé fédérale et dans cette optique, nous nous sommes parfaitement positionnés. Si nous perdons cela de vue et que nous renonçons aux perspectives à long terme pour nous limiter à des objectifs à court terme, nous serons à nouveau bientôt le jouet des politiques. Mais je ne voudrais pas être mal compris: il y a des objectifs à court terme importants, par exemple les questions tarifaires. Mais il y a d'autres niveaux certainement plus importants encore pour l'avenir du corps médical dans la société, qu'il ne faut pas oublier.

En tant que président de la FMH, vous aviez pour mission de trouver un dénominateur commun au corps médical pour les questions importantes de politique de la santé et de le représenter avec conviction envers l'extérieur. Après huit ans, diriez-vous que cette vision du mandat était trop idéaliste par rapport à la réalité?

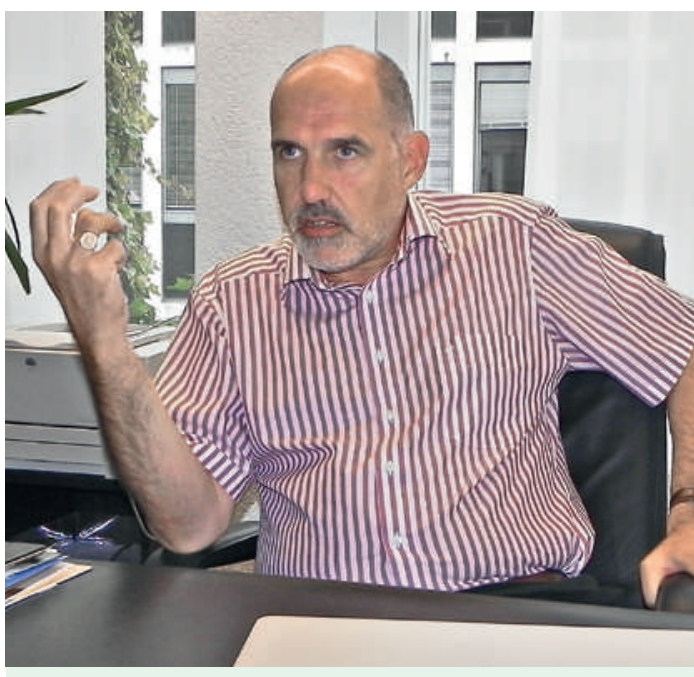

Pour Jacques de Haller, le bilan est positif: «L'évolution des dernières années est claire, nous sommes devenus l'acteur principal du système de santé.»
Il est essentiel que le corps médical reste soudé au sein de la FMH. Si chaque sous-groupe mène son propre combat, qu'il s'agisse de tarif ou d'autre chose, nous perdrons beaucoup d'influence et de possibilités de nous impliquer. Devenir un objet politique plutôt qu'un sujet n'est certainement pas ce que je souhaite à la FMH! Il faut une certaine unité, un certain centralisme. Il faut avoir une vision, il faut savoir ce que l'on veut, il faut pouvoir justifier les choses, même envers soi-même.

Pensez-vous que les intérêts communs pourront compenser également sur le long terme les tendances centrifuges? La FMH a-t-elle encore un avenir?

Le débat sur les tendances à la scission n'est pas nouveau. Lorsque j'ai pris mes fonctions, par exemple, il y avait une crise avec les chirurgiens. Huit ans après, je constate que la FMH est toujours présente, et plus unie que jamais. Nous avons dépassé la question des soins intégrés et les chirurgiens sont restés, les médecins de premier recours sont restés, l'ASMAC est restée. Nous avons maintenant presque 36000 membres avec de nombreux dénominateurs communs.

\section{Qui seraient lesquels?}

La liberté thérapeutique, la recherche de la meilleure qualité possible, le libre choix du médecin, la conviction que le patient ou la patiente doit être au cœur du système de santé. Oui, la FMH a un avenir - si nous relevons les défis à venir, qui sont sans aucun doute importants.

\section{Par exemple?}

Je pourrais citer en exemple la médecine de premier recours. Il nous faut plusieurs centaines de millions de francs pour améliorer la situation des médecins de premier recours, et nous ne les trouverons certainement pas auprès de quelques spécialistes. On pourrait espérer régler le problème des coûts supplémentaires pour les médecins de premier recours par le biais du «Masterplan» du Conseil fédéral. Mais si, à la fin, les médecins de premier recours ne bénéficient pas d'un véritable soutien de la FMH, ils auront du mal à se sentir chez eux au sein de la FMH.

Lorsque l'on remet un mandat, il faut bien sûr faire un bilan. Dans ce sens, j'aimerais vous soumettre quelques mots-clés sur des thèmes importants de votre mandat, en vous priant de les commenter brièvement. Evoquons tout d'abord la réforme structurelle de la FMH.

L'ajustement des structures et la création de l'Assemblée des délégués (AD) furent une réussite. Grâce à cette institution, nous obtenons des résultats concrets, ce qui est positif pour la FMH. Dans la mesure où chaque organisation faîtière dispose d'un nombre de délégués qui n'est pas corrélé directement à sa taille, il n'y a pas de surreprésentation de certains groupes, contrairement à ce qui est le cas à la Chambre médicale. 


\section{Plus concrètement?}

A la Chambre médicale, il existe une majorité «naturelle» des médecins de premier recours qui peut devenir problématique si l'on envisage les perspectives de l'ensemble l'association. Le rôle de l'AD, les débats qui peuvent y avoir lieu et le fait que l'on s'y rencontre ont également fait leurs preuves lors du référendum sur le Managed care. Il n'est pas banal, pour une fédé-

\section{«ll faut une certaine unité, un certain centralisme. Il faut avoir une vision, il faut savoir ce que l'on veut.»}

ration comme la FMH, de maîtriser une telle situation, avec les médecins de premier recours dont la position s'opposait à la position officielle de la FMH.

Passons tout de suite au mot-clé Réseaux de soins.

Il est incontestable qu'une bonne coordination des soins est utile. Le projet de loi sur les réseaux de soins était bon jusqu'à ce qu'il soit faussé par l'augmentation de la franchise, entre autres interventions des assureurs auprès du Parlement. En conséquence de quoi, le projet a été rejeté par la FMH, le PS et l'UDC. Désolé, mais arriver en dernière minute avec de telles «combines» est tout simplement inacceptable et n'a pas été apprécié non plus par le peuple souverain. Si nous avons obtenu au total plus de $75 \%$ des voix, cela vient certainement aussi de nos révélations sur ce type de manœuvres.

Quelle est selon vous la situation des soins intégrés, après la votation?

Comme je l'ai dit, fondamentalement, les réseaux et les soins intégrés sont une bonne chose. Toutefois, les réseaux doivent veiller à conserver un fonctionnement éthique totalement transparent. Si des questions critiques se posent et qu'aucune bonne réponse n'y est apportée, il y a un problème. réduits. Il est important que le corps médical reste crédible sur des questions délicates de ce type.

\section{Mot-clé suivant: TARMED:}

Le TARMED est désormais devenu une normalité. Il fonctionne, même si des ajustements sont nécessaires. La révision en cours n'est pas simple, car ses objectifs ont progressivement évolué. Il est impératif d'améliorer d'abord la situation des médecins de premier recours, avant d'aborder les autres problèmes. Si nous voulons tout résoudre à la fois, les médecins de premier recours n'obtiendront rien et les autres certainement non plus.

Que pensez-vous de la neutralité des coûts?

De divers côtés, on essaie d'éliminer l'idée d'une neutralité des coûts, afin de permettre une actualisation du revenu. Une amélioration financière par une révision du TARMED et dans le cadre de TARVISION n'était pas prévue à l'origine. Si nous voulons nous orienter dans cette direction, nous devons pouvoir le justifier de manière convaincante, ce qui n'est pas facile.

\section{Mot-clé DRG:}

La situation est un peu similaire à celle du TARMED il y a huit ans. Mais l'on peut dire que le système fonctionne, ce qui est valable aussi pour la société SwissDRG SA. De façon remarquable, nous y sommes représentés en tant que FMH, même sans être partenaires tarifaires, ce qui est un gros avantage. Mais nous devons suivre très attentivement l'évolution du système des DRG, notamment avec une recherche concomitante sérieuse. C'est le seul moyen de réagir précocement à des risques de dérapage.

\section{Mot-clé Lobbying:}

Pour le corps médical, il est essentiel que ses intérêts soient représentés auprès du Parlement et des autorités. Je pense que nous avons obtenu de très bons résultats dans ce domaine au cours des dernières années. J'ai toujours précisé très clairement et depuis le dé-

\section{«Fondamentalement, les réseaux et les soins intégrés sont une bonne chose. Toutefois, les réseaux doivent veiller à conserver un fonctionnement éthique totalement transparent.»}

Vous évoquez ici les «kick-backs» des spécialistes, qui ont été récemment traités dans les médias?

C'est un exemple, oui. Des «kick-backs», venant aussi de l'industrie pharmaceutique, ce sont des conflits d'intérêts dont les patients ne sont pas informés. Et puis, si des «kick-backs» sont possibles, l'observateur critique peut du coup se demander si les tarifs des spécialistes sont trop élevés et ne pourraient pas être but que pour nous, des critères différents de ceux des autres branches économiques doivent s'appliquer, différents de ceux appliqués aux agriculteurs, à l'industrie pharmaceutique, etc. Notre domaine d'activités concerne des domaines essentiels de l'humanité, il s'agit de maladie et de santé, de qualité de vie, de vie et de mort. Ce ne sont pas des concepts tels que la rentabilité, l'efficacité, le profit ou la concurrence 
qui sont prioritaires, mais bien la personne humaine. Tel est le message fondamental que nous avons toujours communiqué dans le cadre de notre lobbying.

Les partenaires du monde économique actuel prennent-ils une telle attitude au sérieux?

Il a fallu beaucoup de travail pour que ces idées soient comprises au Parlement, mais finalement, elles ont été acceptées. Je peux maintenant aller dans la salle des pas perdus et dire, par exemple: «Désolé, cette solution est peut-être efficace, mais elle ne convient pas car elle porterait atteinte à l'indispensable relation de confiance avec le patient». Désormais, même le Conseil fédéral comprend de tels arguments. Cela implique cependant que nous soyons totalement crédibles. Sinon, nous deviendrions vite ridicules.

\section{«Notre domaine d'activités concerne des domaines}

\section{essentiels de l'humanité (...) Ce ne sont pas des}

\section{concepts tels que la rentabilité, l'efficacité, le profit}

\section{ou la concurrence qui sont prioritaires.»}

\section{Mot-clé Obligation de contracter:}

Dans ce domaine, les débats sont très idéologiques. De grands partis comme le PLR et l'UDC souhaitent encore la suppression de l'obligation de contracter. Manifestement, tout le monde n'a pas encore compris que le libre choix du médecin est essentiel pour la population. C'est sur ce thème que nous avions orienté notre campagne pour la votation sur les réseaux de soins et $76 \%$ de la population nous ont suivis.

\section{Mot-clé Manque de médecins:}

Nous devons rechercher des solutions globales. En effet, les médecins ne sont pas les seuls à être concernés, toutes les professions de la santé le sont. Le problème de la relève n'est pas uniquement un problème d'argent - si les revenus ne sont plus aussi élevés que dans le temps, ils restent néanmoins en général corrects - mais il s'agit aussi de conditions de travail et de qualité de vie.

\section{Mot-clé Médias:}

Nous devons être présents, et dans ce domaine aussi nous avons beaucoup progressé ces dernières années. Nous faisons désormais partie du paysage, nous sommes devenus incontournables. Les journalistes ont besoin d'informations pour faire leur travail, et nous pouvons leur en fournir. Globalement, la collaboration fonctionne parfaitement, et parmi plus de 100 journalistes, j'en connais au plus deux ou trois que j'ai trouvés incorrects.

Conférence suisse des directrices et directeurs cantonaux de la santé

** Association suisse des infirmières et infirmiers
Comment avez-vous vécu la relation avec les partenaires principaux du secteur de la santé?
Bizarrement, jusqu'à l'année dernière, le secteur de la santé était «exclusivement romand». Le conseiller fédéral compétent était un Suisse romand, tout comme les présidents de $\mathrm{H}+$, santésuisse, pharmaSuisse, la CDS* et l'ASI**. Cette configuration allait de pair avec une bonne culture du débat, même si nos opinions concrètes divergeaient souvent. Mais en réalité, les relations dépendent toujours des personnes.

Pouvez-vous citer quelques exemples concrets?

Nous n'avons par exemple jamais réussi à avoir un dialogue constructif, avec le conseiller fédéral Pascal Couchepin. Avec lui, il y avait toujours des problèmes de pouvoir. En revanche, la collaboration avec Didier Burkhalter a été bonne, même si sa motivation a semblé faiblir après quelques mois. Nous travaillons aussi très bien avec Alain Berset et son équipe. Il en va de même pour santésuisse: mes relations avec son actuel directeur, Christoph Meyer, sont excellentes.

A votre avis, quels sont les questions et problèmes les plus urgents auxquels le corps médical sera confronté dans un avenir plus ou moins proche?

Dans un avenir proche, je citerais l'initiative des médecins de famille et la caisse unique. Je ne sais pas encore quelle position la FMH adoptera pour la question de la caisse unique, mais il sera essentiel que la base puisse s'exprimer sur le sujet dans le cadre d'un vote général. Sinon, de fortes tensions sont à prévoir.

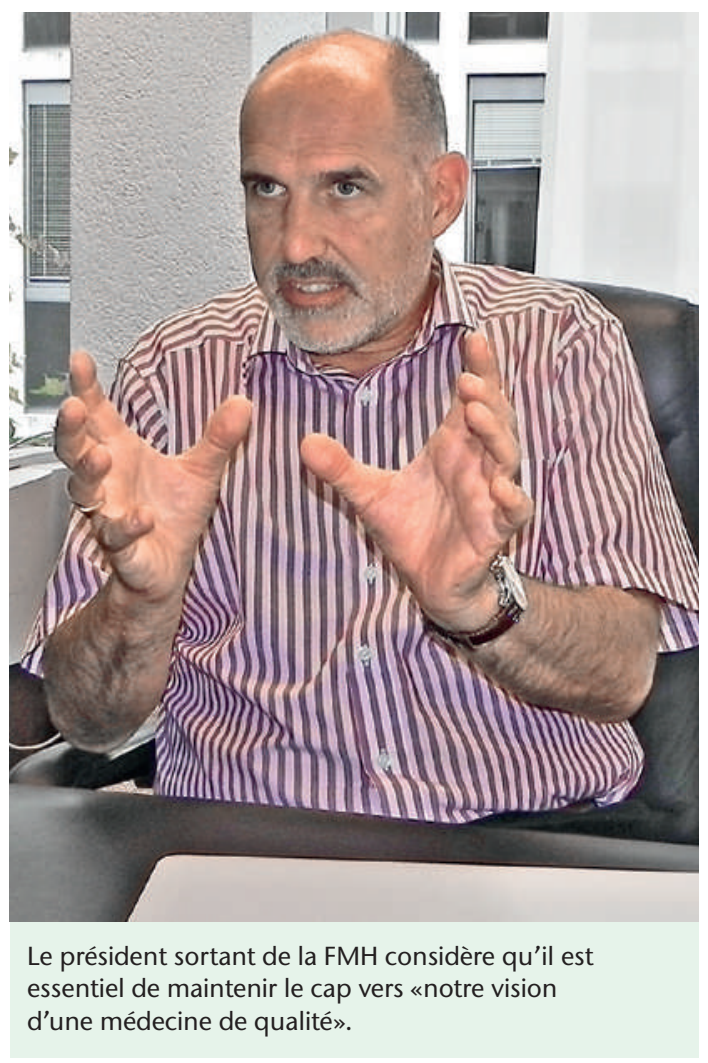




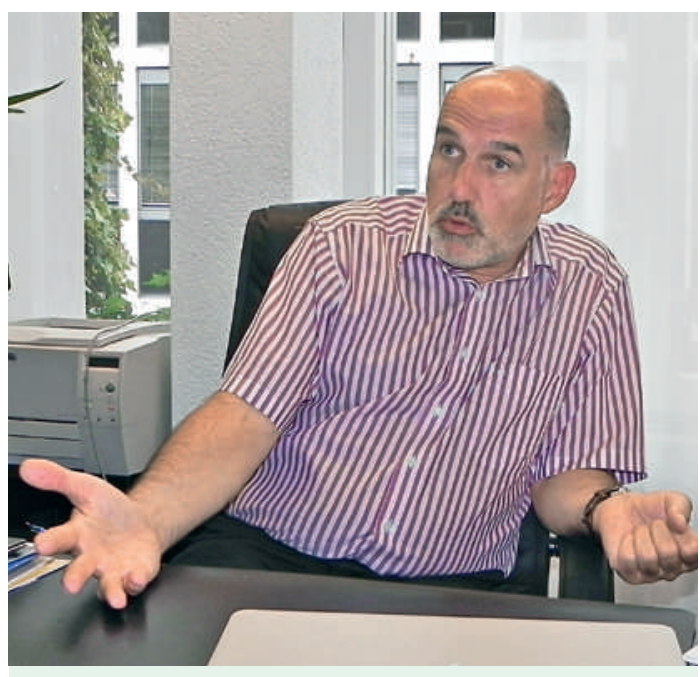

Aucun signe d'usure: Jacques de Haller se sent aujourd'hui encore plus en forme qu'au moment où il a pris ses fonctions.

Quelles sont les perspectives à plus long terme?

A long terme, le financement du système de santé constituera certainement un problème important, mais là, l'essentiel pour moi est que nous ne considérions pas seulement nos tarifs, mais aussi notre vision d'une bonne médecine. Nous devons systématiquement défendre ces points de vue larges, en politique. A défaut, nous serons bientôt marginalisés et nous n'aurons plus rien à dire.

\section{«Lorsque l'on aborde un mandat de ce type, il faut}

l'avoir voulu, y avoir bien réfléchi, connaître les implica-

tions. En effet, au niveau de la personne, les exigences sont très fortes.»

Qu'entendez-vous par «notre vision d'une bonne médecine»?

Chaque année, un médecin conduit 2000 et 5000 consultations. En 20 ans, cela peut correspondre à près de 100000 rencontres. Il en résulte un incroyable capital d'expérience humaine et, espérons-le, une vision humaniste de la personne et du monde. Ne pas intégrer tout cela au débat socio-politique, se limiter uniquement aux aspects purement techniques de la médecine serait vraiment dommage.

Vous avez ainsi passé à un niveau un peu plus personnel. Quels domaines de votre activité de président de la FMH avez-vous ressentis comme particulièrement enrichissants? En soi, la variété du travail représente bien sûr un enrichissement: un telle palette d'activités est toujours passionnante. J'ai ressenti aussi très positivement les innombrables contacts personnels avec des personnes très différentes, les échanges directs, et également d'avoir à convaincre.

\section{Et quels ont été les aspects négatifs?}

J'ai surtout trouvé très dérangeants les préjugés auxquels j'étais régulièrement confronté et qui émanaient le plus souvent de personnes actives en politique professionnelle. Et puis, il y a les manœuvres politiques, les intérêts personnels mis au premier plan et les appétits de pouvoir qui finissent par paralyser beaucoup une fédération comme la FMH. Lorsque la FMH développe plus de pouvoir et de reconnaissance, c'est considéré comme une menace, dans ces cercles, qui bloquent alors tout pour sauvegarder leurs propres domaines d'influence. Malheureusement, je ne pense pas que cela va s'améliorer ces prochaines années, mais la FMH va devoir une fois se confronter à cet état de fait.

Il va de soi que votre mandat implique une charge importante. Sentez-vous certains signes d'usure?

Je n'ai vraiment pas le sentiment d'être «au bout du rouleau». Lorsque l'on aborde un mandat de ce type, il faut l'avoir voulu, y avoir bien réfléchi, en connaître les implications. En effet, au niveau de la personne, les exigences sont très fortes. Il est politiquement incorrect, mais réel, que je n'ai jamais fait de séparation nette entre mon travail et les loisirs. Ce n'était tout simplement pas possible. Le fait de travailler à $200 \%$ ne m'a à aucun moment posé problème. J'ai beaucoup aimé être président de la FMH, et je n'avais simplement pas besoin d'un hobby supplémentaire. Comme je l'ai dit, cela peut sembler incorrect, mais c'est la vérité. D'ailleurs, je suis en meilleure forme que lorsque j'ai pris mes fonctions, par exemple, je fais plus de sport.

Comment les générations futures définiront-elles «l'ère de Haller», quels aspects resteront dans les mémoires?

C'est une question délicate. Aujourd'hui, je citerais le positionnement de la FMH dans la politique nationale, le fait que nous sommes devenus le lobby le plus efficace de Suisse (cette estimation vient d'un journal alémanique très connu). Il s'agissait là pour moi d'un objectif prioritaire, et cela décrit probablement le mieux cette période.

Avez-vous déjà des idées des domaines dans lesquels vous souhaitez vous engager à l'avenir? Resterez-vous acquis au système de santé, à la politique de santé, ou souhaitezvous vous réorienter et vous engager dans une direction totalement différente?

Ce sera certainement le domaine du système et de la politique de santé, dans lequel je dispose désormais d'une grande expérience et d'un bon réseau. Ce serait dommage de laisser ces atouts inutilisés, d'autant plus que ce secteur d'activités me fascine toujours. Je ne sais pas du tout ce que je ferai précisément, mais cela n'est pas un souci! 